\title{
Different CT Findings of Aortic Hemorrhage Extending to Pulmonary Artery from Stanford Type A Aortic Dissection
}

\author{
Kyu-Chong Lee, ${ }^{1}$ Jae Wook Lee, ${ }^{1, *}$ Hwan Seok Yong, ${ }^{1}$ and Eun-Young Kang1 \\ ${ }^{1}$ Department of Radiology, College of Medicine, Korea University, Seoul, Korea \\ "Corresponding author: Jae Wook Lee, Department of Radiology, College of Medicine, Korea University, Seoul, Korea. Tel: +82-1029235431, Fax: +82-28639282, E-mail: \\ ljw2000e@naver.com
}

Received 2017 May 17; Revised 2017 November 13; Accepted 2018 January 23.

\begin{abstract}
Hemorrhage extending to the pulmonary artery is an uncommon complication of Stanford type A aortic dissection that has a poor prognosis. Diagnosis of this complication is mainly dependent on imaging studies, especially computed tomography. High attenuation can be seen along the wall of the pulmonary artery on the non-enhanced study, while the enhanced study shows luminal narrowing and wall thickening of the pulmonary artery. We report three cases with different imaging findings of hemorrhage extending to the pulmonary artery from Stanford type A aortic dissection.
\end{abstract}

Keywords: Hemorrhage Extending to the Pulmonary Artery, Aortic Dissection, Computed Tomography

\section{Introduction}

Hemorrhage extending along the pulmonary artery from the ruptured aorta is an uncommon complication of Stanford type A aortic dissection. It can occur when the posterior wall of an aorta is ruptured because the pulmonary trunk and ascending aorta have a common adventitia $(1,2)$. Only a few reports were published previously on this subject (2-6). It can be diagnosed by an imaging study such as computed tomography (CT).

We report three cases of hemorrhage extending to the pulmonary artery from Stanford type A aortic dissection and review general features, diagnosis and management of this complication.

\section{Case Presentation}

\subsection{Case 1}

An 87-year-old female patient arrived at our emergency department with general weakness and dizziness. She had a history of hypertension, and electrocardiography (ECG) showed atrial fibrillation. Chest radiography showed cardiomegaly with bulging opacity in the right hilar area. Cardiac biomarkers such as creatine kinase muscle and brain (CK-MB) (4.15 ng/mL), pro-B-type natriuretic peptide (BNP) (151.37 pg/mL), and creatine phosphokinase (CPK) (210 IU/L) were elevated, and echocardiography was performed to evaluate heart function. Findings suggested the presence of an ascending aortic aneurysm with intramural hematoma and penetrating ulcer. No regional wall motion abnormality was seen in echocardiography. Consequently, an ECG-gated CT aortography was performed on a 64 detector-row CT scanner (Brilliance 64, Philips Medical Systems, Cleveland, OH, USA). CT revealed a Stanford type A dissection involving the aortic root with saccular aneurysmal dilatation (maximal diameter $=8.5 \mathrm{~cm}$ ). Aortic dissection did not involve coronary arteries. On contrastenhanced images, wall thickening and luminal narrowing of the main and right pulmonary arteries were observed. Corresponding region showed high attenuation on nonenhanced images that indicated a hemorrhage. Furthermore, in lung window setting, interstitial thickening and ground-glass opacity in the lung parenchyma suggested hemorrhage extension into the alveoli (Figure 1). Unfortunately, while waiting for emergency surgery, the patient's blood pressure and heart rate dropped, and she eventually died even after vigorous resuscitation efforts.

\subsection{Case 2}

A 72-year-old female patient visited our emergency department with complaints of right thigh pain and mild chest discomfort. She had a history of hypertension, and an initial chest radiography showed mild cardiomegaly. Cardiac enzyme levels were normal. Lower extremity CT arteriography was performed due to pulseless and pale 

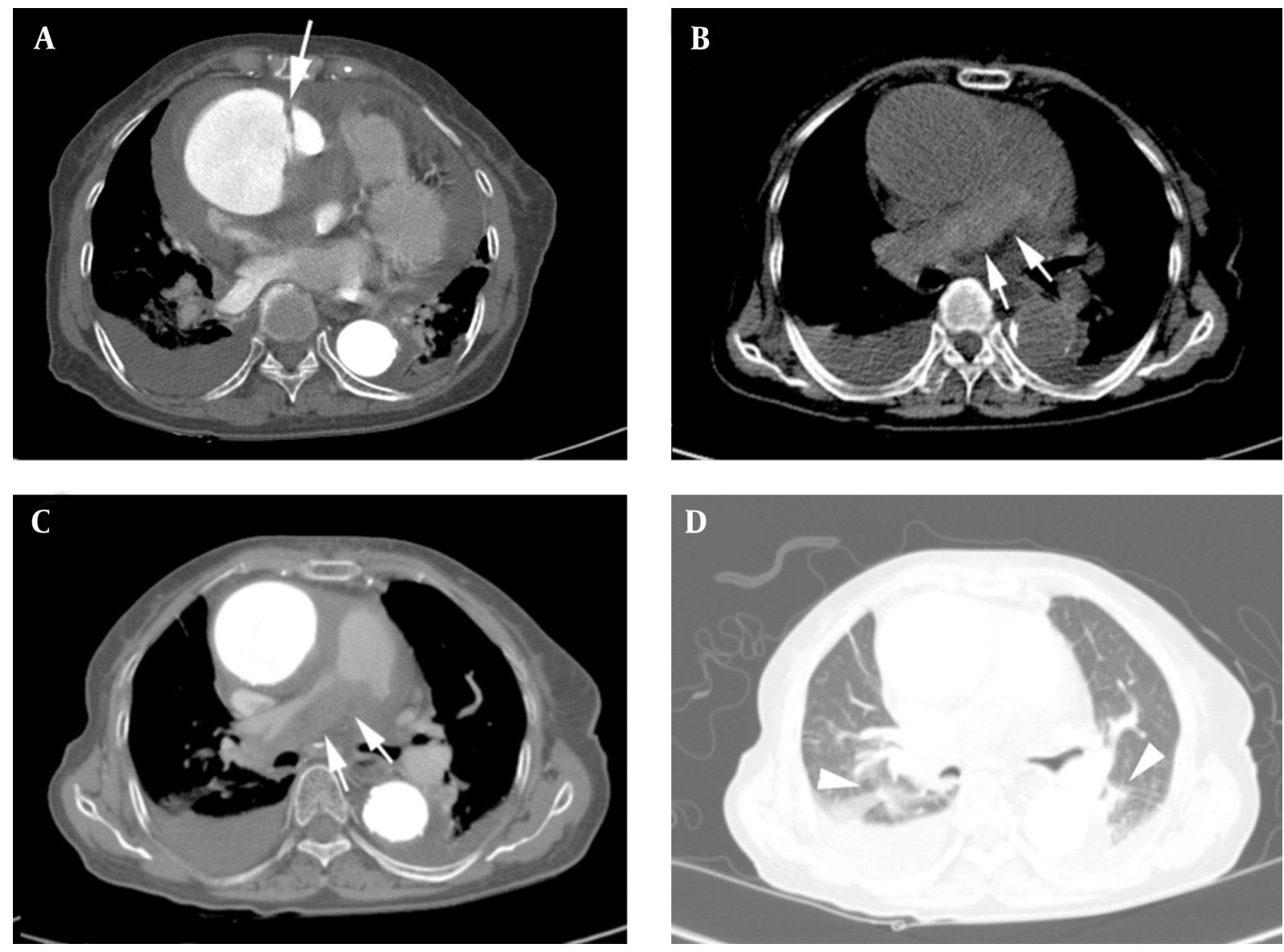

Figure 1. An 87-year-old woman who complained of general weakness and dizziness. A, Axial enhanced CT image in a mediastinal window setting demonstrates a dissection flap (arrow) in the proximal ascending aorta with aortic aneurysm. B, Axial non-enhanced CT image in a mediastinal window setting demonstrates hemorrhage along the main and right pulmonary artery as high attenuation (arrows). C, Axial enhanced CT image in a mediastinal window setting demonstrates wall thickening with severe luminal narrowing (arrows). D, Axial enhanced CT image in a mediastinal window setting demonstrates interstitial thickening and ground-glass opacity in lung parenchyma suggest hemorrhage extension into the alveoli (arrowheads).

dorsum of the right foot. CT scan revealed aortic dissection from the abdominal aorta to both common iliac arteries. Right proximal common iliac artery was compressed by thrombus filled false lumen. To evaluate the origin of aortic dissection, an ECG-gated CT aortography was performed 40 minutes after lower extremity CT arteriography. On enhanced CT, dissection of the aortic root along with wall thickening and luminal narrowing of the pulmonary artery were observed. This suggested that the hemorrhage from the aortic dissection extended to the pulmonary artery. However, unlike the previous case, we could not distinguish the high attenuation along the pulmonary arterial wall from the pulmonary arterial lumen on pre-contrast CT. This was because previously injected contrast media from the lower extremity CT arteriography had not completely washed out, which resulted in high attenuated lumen on pre-contrast CT (69 Hounsfield unit). In addition, ground glass opacity was seen on the right central lung, suggesting hemorrhage extension to the alveoli in lung window setting (Figure 2). Blood pressure and heart rate continued to fall while waiting for emergency surgery. Despite careful management, the patient died on the following day.

\subsection{Case 3}

An 86-year-old female patient arrived at our emergency department with acute onset chest pain radiating to the back and mild dyspnea. She had a history of hypertension, and initial vital signs were stable. Electrocardiography suggested left ventricular hypertrophy. Cardiac enzyme levels were normal. D-dimer was elevated (3.36 ug/mL). Initial chest radiography showed dilated and tortuous aortic shadowing along with mild cardiomegaly. A pulmonary embolism CT scan was further performed to evaluate the 

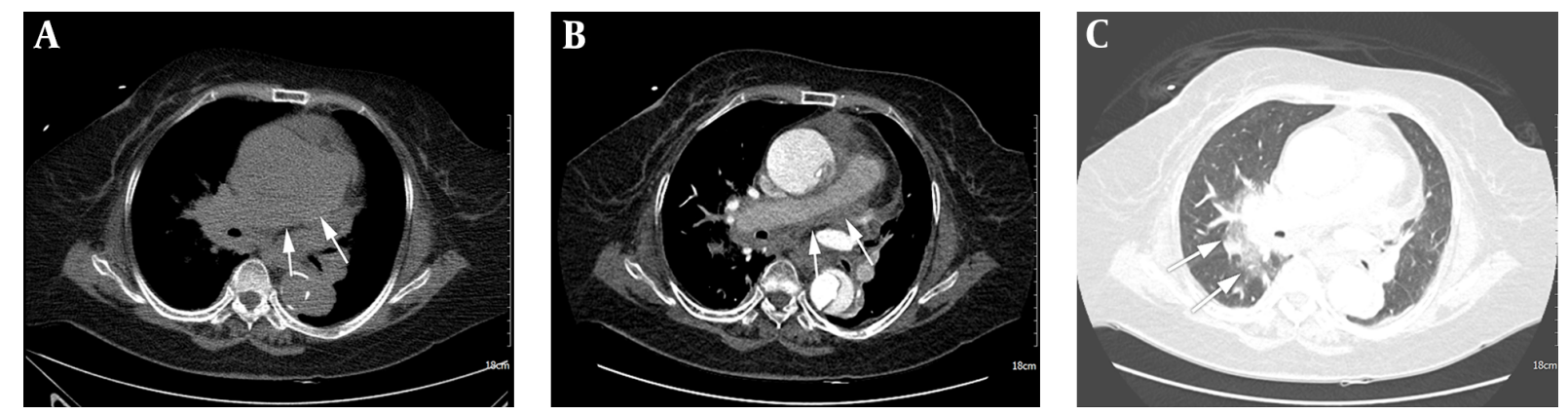

Figure 2. A 72-year-old woman who complained of right thigh pain and mild chest discomfort. A, Axial non-enhanced CT image in a mediastinal window setting. Hemorrhage is concealed due to previously injected contrast media, resulting in high attenuated lumen (69 Hounsfield unit). B, Axial enhanced CT image in a mediastinal setting. Wall thickening and luminal narrowing of the pulmonary artery suggest hemorrhage from ruptured aorta extending to the pulmonary artery (arrows). C, Axial enhanced CT image in a lung window setting. Ground glass opacity in the right central lung suggests hemorrhage extension to the alveoli (arrows).

patient's symptom and D-dimer elevation. There was no pulmonary embolism, but Stanford type A aortic dissection from the aortic root was noted. In addition, narrowing of both pulmonary arteries indicated that these findings together suggested extension of hemorrhage from aortic dissection to both pulmonary arteries. However, we were not able to evaluate high-attenuated hemorrhage because non-enhanced images were not included in our routine pulmonary embolism CT protocol. On lung window setting, patchy ground glass opacity in the right upper and central lung suggested hemorrhage extension to the alveoli (Figure 3). Emergency operation was considered but was not performed due to patient's old age and poor general condition. The patient was transferred to a nursing hospital for conservative management.

\section{Discussion}

Aortic dissection is the most common cause of aortic emergency and has a high mortality rate (7). Therefore, rapid diagnosis and management are necessary. Fortunately, recent advancement in imaging techniques, such as ECG-gated CT aortography and MRI, contributes to diagnosing acute aortic dissection more rapidly and easily. CT is the preferred modality over MRI in emergency situations due to shorter scanning time. This imaging modality not only differentiates aortic dissection types but is also capable of evaluting fatal complications of aortic dissection such as hemopericardium, hemothorax, and organ ischemia (7).

Among those complications, hemorrhage extending along the pulmonary artery is an uncommon complication of Stanford type A aortic dissection. This complication reduces pulmonary circulation, which eventually decreases left ventricle preload resulting in a devastating outcome. Only a few reports were published previously (2-
6). Sueyoshi et al. found 21 cases ( $9 \%$ ) of 232 patients with Stanford A aortic dissection (2), but the true prevalence of this complication is still unclear because this complication is probably under-recognized. Anatomically, pulmonary trunk and ascending aorta have a single common adventitia at the roots of the great vessels, and the ascending aorta is anterior to the pulmonary artery at the roots of the great vessels. Therefore, hemorrhage resulting from the rupture of the posterior wall of the aorta can extend into the space between the media of the pulmonary artery and common adventitia. This can compress and narrow the pulmonary arterial lumen as the aortic pressure is higher than the pulmonary artery pressure $(1,2)$.

Diagnosis of this complication is mainly dependent on imaging studies. According to previous reports, chest radiographic findings are variable from normal to mimicking pulmonary edema. Transthoracic echocardiography can detect aortic dissections and some complications such as hemopericardium (8), however, it has limitations when it comes to evaluating the pulmonary artery. As a result, CT is the most sensitive method to evaluate the complication of aortic dissection. On nonenhanced CT, hemorrhage is visible as high attenuation along the wall of the pulmonary artery. On enhanced CT, it is usually interpreted as pulmonary arterial wall thickening with narrowed lumen (2-4). Accurate diagnosis is guaranteed only when both pre and post contrast enhanced scans are obtained. For example, our first case showed wall thickening and luminal narrowing of the pulmonary artery on enhanced CT, and the wall thickening was confirmed as hemorrhage by high attenuation in the corresponding region on non-enhanced CT. However, not all cases reveal both findings. In our second case, pre-contrast CT was performed just after lower extremity CT angiography, so highly attenuated hemorrhage was concealed by previously injected contrast-media. On 

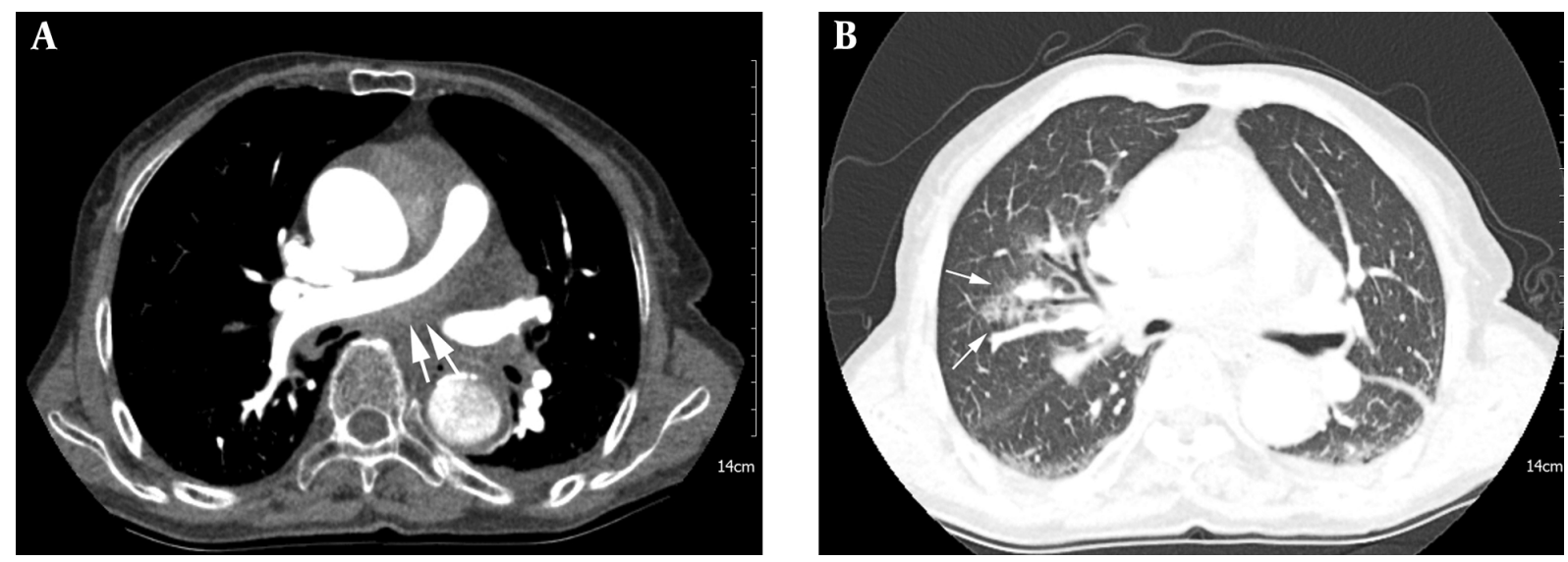

Figure 3. An 86-year-old woman who had acute onset chest pain radiating to the back and mild dyspnea. A, Axial enhanced CT image in a mediastinal window setting. Hemorrhage from the ruptured aorta extends along both pulmonary arteries resulting in narrowing of both pulmonary arteries (arrows). B, Axial enhanced CT image in a lung window setting. Patchy ground glass opacity in the right upper and central lung suggests hemorrhage extension to the alveoli (arrows).

the other hand, sometimes contrast media from a previous study remains in the aortopulmonary adventitia and is seen as a high density lesion. This should be differentitated from hemorrhage (6). Therefore, if any contrastenhanced CT was performed before CT aortography, physicians should consider the effect of previously injected contrast media. Furthermore, like in our third case, nonenhanced CT images are not always routinely included in CT scans. Therefore, in these situations, enhanced images should be more carefully evaluated.

Sueyoshi et al. (2) classified this complication into three catergories based on the extent of hemorrhage shown by the CT image. The first category includes abnormal density limited only to the pulmonary artery, indicating hemorrhage is localized around the pulmonary artery. The second category includes the presence of interlobular septal thickening on CT, which indicates hemorrhage extension into the interlobular septa. The third category is defined as hemorrhage extension into the alveoli, and the CT image shows ground-glass opacity in the affected lung field. Higher category is associated with worse prognosis (2). Therefore, we also have to carefully assess lung images. All three of our cases can be classified into the third category.

Stanford type A aortic dissection itself has a poor prognosis, but when it is combined with complications, the prognosis is even worse. Hemorrhage extending to the pulmonary artery is one of the most fatal complications of Stanford type A aortic dissection and emergent surgical management needs to be considered (9). Therefore, a quick and accurate imaging evaluation can help the patient receive appropriate management. Typical imaging findings of a hemorrhage extending to the pulmonary ar- teries are noted by high attenuation along the wall of the pulmonary artery on nonenhanced CT, while enhanced CT images show wall thickening and luminal narrowing of the artery. However, not all cases reveal both findings and different CT findings can make diagnosis difficult. Therefore, we should keep this complication in mind when aortic dissection is considered.

\section{Footnotes}

Authors' Contributions: None declared.

Financial Disclosure: None declared.

Funding/Support: None declared.

\section{References}

1. Roberts WC. Aortic dissection: anatomy, consequences, and causes. Am Heart J. 1981;101(2):195-214. doi: 10.1016/0002-8703(81)90666-9. [PubMed: 7008565].

2. Sueyoshi E, Matsuoka Y, Sakamoto I, Uetani M. CT and clinical features of hemorrhage extending along the pulmonary artery due to ruptured aortic dissection. Eur Radiol. 2009;19(5):1166-74. doi: 10.1007/s00330-008-1272-7. [PubMed: 19156424].

3. Cao DB, Yang SR, Tong Q, Zheng Y. Interstitial pulmonary hemorrhage along the pulmonary artery secondary to ruptured aortic dissection. Intern Med. 2010;49(15):1681-2. doi: 10.2169/internalmedicine.49.3874. [PubMed: 20686318].

4. Sueyoshi E, Sakamoto I, Uetani M, Matsuoka Y, Suenaga E. CT findings of ruptured intramural hematoma of the aorta extending along the pulmonary artery. Cardiovasc Intervent Radiol. 2007;30(2):321-3. doi: 10.1007/s00270-005-0388-2. [PubMed: 16988873].

5. Panicek DM, Ewing DK, Markarian B, Heitzman ER. Interstitial pulmonary hemorrhage from mediastinal hematoma secondary to aortic rupture. Radiology. 1987;162(1 Pt 1):165-6. doi: 10.1148/radiology.162.1.3786757. [PubMed: 3786757]. 
6. Guilmette J, Semionov A, Dennie C, Gahide G, Pressacco J, Fraser R, et al. Hemorrhagic infiltration of the aortopulmonary adventitia: A complication of acute aortic dissection. EurJ Radiol.2016;85(1):239-47. doi: 10.1016/j.ejrad.2015.11.025. [PubMed: 26724672].

7. Castaner E, Andreu M, Gallardo X, Mata JM, Cabezuelo MA, Pallardo Y. CT in nontraumatic acute thoracic aortic disease: typical and atypical features and complications. Radiographics. 2003;23 Spec No:S93-110. doi: 10.1148/rg.23si035507. [PubMed: 14557505].
8. Mohr-Kahaly S, Epbel R, Kearney P, Puth M, Meyer J. Aortic intramural hemorrhage visualized by transesophageal echocardiography: Findings and prognostic implication. J Am Coll Cardiol. 1994;23(3):658-4. doi: 10.1016/0735-1097(94)90751-x.

9. Nienaber CA, von Kodolitsch Y, Petersen B, Loose R, Helmchen U, Haverich A, et al. Intramural hemorrhage of the thoracic aorta. Diagnostic and therapeutic implications. Circulation. 1995;92(6):1465-72. doi: 10.1161/01.CIR.92.6.1465. [PubMed: 7664428]. 